\title{
Metabolic Mechanisms in Obesity and Type 2 Diabetes: Insights from Bariatric/Metabolic Surgery
}

\author{
Adriana Florinela Cătoi ${ }^{a} \quad$ Alina Pârvu $^{a} \quad$ Adriana Mureşan $^{a} \quad$ Luca Busetto $^{b}$ \\ aDepartment of Functional Biosciences, Faculty of Medicine, 'Iuliu Haţieganu' University of \\ Medicine and Pharmacy, Cluj-Napoca, Romania; ${ }^{b}$ Department of Medicine, University of \\ Padova, Padova, Italy
}

\section{Key Words}

Obesity · Type 2 diabetes · Metabolic surgery · Hyperinsulinemia · Insulin resistance ·

Surgical food restriction $\cdot$ Incretins

\begin{abstract}
Obesity and the related diabetes epidemics represent a real concern worldwide. Bariatric/ metabolic surgery emerged in last years as a valuable therapeutic option for obesity and related diseases, including type 2 diabetes mellitus (T2DM). The complicated network of mechanisms involved in obesity and T2DM have not completely defined yet. There is still a debate on which would be the first metabolic defect leading to metabolic deterioration: insulin resistance or hyperinsulinemia? Insight into the metabolic effects of bariatric/metabolic surgery has revealed that, beyond weight loss and food restriction, other mechanisms can be activated by the rearrangements of the gastrointestinal tract, such as the incretinic/anti-incretinic system, changes in bile acid composition and flow, and modifications of gut microbiota; all of them possibly involved in the remission of T2DM. The complete elucidation of these mechanisms will lead to a better understanding of the pathogenesis of this disease. Our aim was to review some of the metabolic mechanisms involved in the development of T2DM in obese patients as well as in the remission of this condition in patients submitted to bariatric/metabolic surgery.
\end{abstract}

(c) 2015 S. Karger GmbH, Freiburg

\section{Introduction}

Obesity has become a real concern worldwide due to its increasing prevalence and to the associated cluster of diseases that reduce life quality and expectancy. Patients with BMI over $40 \mathrm{~kg} / \mathrm{m}^{2}$ as well as those with a BMI over $35 \mathrm{~kg} / \mathrm{m}^{2}$ and comorbidities, who have failed to 
Cătoi et al.: Metabolic Mechanisms in Obesity and Type 2 Diabetes: Insights from Bariatric/Metabolic Surgery

obtain and maintain significant weight reduction through nonsurgical means, are submitted to bariatric surgery in order to reach the weight goals [1]. Type 2 diabetes mellitus (T2DM) is the major comorbidity of obesity, and therefore the terms 'diabesity' and 'obesity-dependent diabetes' are often used [2]. Approximately half of the individuals diagnosed with T2DM are obese [2].

Bariatric surgery exerts a complex impact on human body, beyond weight loss, by improving metabolic health. Therefore, the term 'metabolic surgery' recently has been proposed as a more appropriate labelling [3, 4]. The term 'metabolic surgery' was used for the first time 35 years ago by Richard L. Varco and Henry Buchwald [5], and it was that time defined 'as the operative manipulation of a normal organ or organ system to achieve a biological result for a potential health gain'. Indeed, a large body of evidence has demonstrated that several gastrointestinal operations primarily designed to promote weight loss can ameliorate or restore T2DM $[6,7]$. Bariatric/metabolic surgery has been found to be more effective than conventional medical therapy in inducing T2DM improvement or remission in several randomized and non-randomized controlled trials [8-12]. It is reasonable to suppose that surgical weight loss per se is associated with remission of T2D M. However, rapid improvements in blood glucose levels, occurring within days after surgery long before substantial weight loss, initially suggested that other mechanisms beyond weight reduction could be involved in these changes [13]. As explained below, this was not the case, giving that such initial improvements may be more simply explained by postoperative caloric restriction, but some possible non-weight-loss-related mechanisms are probably involved in graduating the metabolic response to different surgical procedures and in maintaining these improvements over time. The details have not yet completely understood, but a more complete elucidation of the metabolic changes induced by bariatric/metabolic surgery may also bring light upon the pathogenesis of T2DM in patients with obesity. Some of the mechanisms involved in the development of T2DM in obesity as well as in T2DM remission after bariatric/metabolic surgery will be reviewed here.

\section{Obesity and Type 2 Diabetes}

\section{Insulin Resistance or Hyperinsulinemia: Who Is Coming First?}

T2DM is a heterogeneous disorder most commonly characterized by insulin resistance, a state of reduced insulin-mediated glucose uptake, in the presence of incapacity of the pancreatic beta cells to produce and provide sufficient insulin to meet the required needs [14]. T2DM may range from insulin resistance with relative insulin deficiency to a secretory defect with minimal insulin resistance. The classical view for explaining the development of T2DM in obesity states that insulin resistance is the first lesion, followed by hyperinsulinemia, and that hyperglycemia occurs as the consequence of beta cell failure to produce enough insulin. Insulin resistance in muscle is caused by inherited and environmental factors and is the earliest detectable disturbance of T2DM [15-17]. T2DM is a disease that progresses despite glucose-lowering medication, and $50 \%$ of the patients require insulin therapy within 10 years [18].

More recently, Taylor et al. $[15,16]$ proposed the 'twin cycle hypothesis' to better explain the etiology of T2DM in obese patients (fig. 1). Whole-body insulin resistance is the earliest predictor of T2DM onset [19]. However, hepatic rather than muscle insulin resistance is involved in the occurrence of hyperglycemia and overt diabetes [20]. The pre-existing muscle insulin resistance caused by inherited and environmental factors leads to raised plasma insulin levels, which, together with an increased caloric overload, will exert accumulation of fat in the 
Fig. 1. Muscle insulin resistance and the 'twin cycle hypothesis': roles of muscle, liver and pancreas in the occurrence of T2DM in obesity.

Cătoi et al.: Metabolic Mechanisms in Obesity and Type 2 Diabetes: Insights from Bariatric/Metabolic Surgery

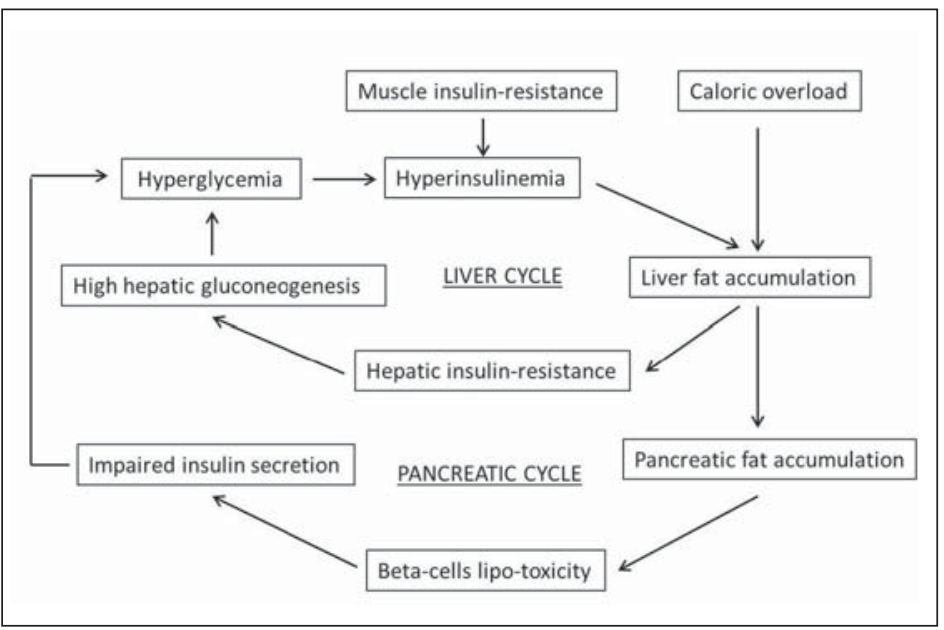

liver. In turn, liver fat accumulation causes hepatic insulin resistance, hence the failure of insulin to suppress liver glucose production. Consequently, increased plasma glucose as well as further increased basal insulin secretion will develop, exacerbating hepatic steatosis. This forms the first vicious cycle (the liver cycle). Liver fat infiltration, and related high fatty acid turnover, then induces an over-exposure of the beta cells to fatty acids, which will impair the acute insulin secretion in response to ingested food and lead to postprandial hyperglycemia. Hyperglycemia will further stimulate insulin secretion which will consequently increase liver lipogenesis, thus connecting the liver cycle with a new vicious cycle (the pancreas cycle). Noteworthy, fatty liver is a disturbance that is present years before the T2DM development. A history of 2-3 years of T2DM does not produce irreversible damages to the beta cells, but as long as the energy overload persists through years, irreversible impairment of the beta cells occurs, and insulin is required in order to control plasma glucose $[15,16]$. As outlined below, this more complex metabolic view may be of great relevance in explaining the rapid improvements in hyperglycemia observed after bariatric/metabolic surgery.

On the other hand, Pories et al. [17] proposed an alternative hypothesis to explain the cause of T2DM in obesity: Fasting hyperinsulinemia would be the initial alteration, and the remission of the disease following bariatric surgery may be due to the correction of hyperinsulinemia [17]. The authors hypothesized that a putative diabetogenic signal could have been released from the gastrointestinal tract in response to overfeeding and that this factor may induce fasting hyperinsulinemia, which in turn blunts the normal insulin response to a meal. In spite of insulin resistance, the continuous elevated basal insulin levels determine the muscle to take up more glucose than needed or able to be stored. Once in the muscle, glucose will have converted into pyruvate with subsequent conversions to lactate and alanine. These metabolites represent gluconeogenetic substrates that will have released from the muscle and, once reached the liver, will lead to an increased hepatic production of glucose. The high levels of fasting glucose and lactate in diabetic patients could be then defined to be a consequence of a 'vicious Cori Cycle' [17].

\section{Impairment of the Entero-Insular Axis}

Unger and Eisentraut [21] invented in 1969 the concept of the entero-insular axis, which refers to the link between the gut and the pancreatic islets. Later, Creutzfeldt [22] developed a concept that the axis encompasses nutrient, hormonal and neural signals from the gut to the 
Cătoi et al:: Metabolic Mechanisms in Obesity and Type 2 Diabetes: Insights from Bariatric/Metabolic Surgery

beta cells, and defined the hormonal or incretinic part of the entero-insular axis. Incretins are gut hormones that are released by the gastrointestinal cells upon the contact with nutrients, mainly carbohydrates. At physiological levels these nutrients stimulate insulin secretion in the presence of elevated blood glucose levels [21]. Glucose-dependent insulino-tropic polypeptide (GIP) and glucagon-like polypeptide-1 (GLP-1) are the two hormones that meet the criteria for incretin definition [23-25]. GLP-1 is produced by the endocrine L cells from the terminal ileum and colon. GLP-1 induces secretion and production of insulin and has been shown to significantly improve postprandial glycemic control [26]. GLP-1 upregulates pancreatic beta cell genes, promotes beta cell proliferation and inhibits beta cell apoptosis [27, 28]. Beyond the incretinic effect, GLP-1 inhibits glucagon secretion and gastric emptying $[29,30]$ and exerts metabolic actions by increasing glyconeogenesis in the liver and skeletal muscle and lipogenesis in adipose tissue [26, 29]. GIP is released by the K-type endocrine cells that are clustered in the duodenum and proximal jejunum as a result of carbohydrate and fat absorption $[25,26]$. It seems that the main stimulus for GIP release is the rate of absorption, and not the luminal content [25]. GIP induces glucose-mediated insulin secretion but also stimulates beta cell proliferation and inhibits beta cell apoptosis [29]. It has been demonstrated that $80 \%$ of the entero-insular effect on insulin secretion is driven by GIP [31]. GIP acts on the adipose tissue as well, stimulating lipoprotein lipase activity and incorporation of fatty acids [23].

Obesity and T2DM are associated with impairment of the entero-insular axis, defined as a reduced capacity of incretins to induce glucose-mediated insulin secretion as a result of a meal ingestion $[23,24]$. Impairment of GLP- 1 action is caused by a blunted secretion of $L$ cells, as observed in early stages of T2DM [32]. Some studies demonstrated that in patients with T2DM there is also a reduced secretion of GLP-1 under basal conditions as well as postprandially $[33,34]$. However, with respect to GIP, it seems that chronic desensitization and downregulation of the specific receptors on the pancreatic beta cells are the main incriminating factors [23]. Indeed, GIP levels appear to be normal or slightly elevated in patients with obesity and T2DM, both in basal and post-prandial states [35]. GLP-1 and GIP account for approximately $50 \%$ of the insulin secretion after a meal, thus implying that the impaired incretinic effect in T2DM plays an important role in the development of this disease [36].

The etiologic mechanism proposed by Pories [37] and Pories et al. [38] for the development of T2DM development in obese patients implies that metabolic deterioration progress as a result of an overstimulation of incretins induced by overeating, leading to hyperinsulinemia and insulin resistance. However, this hypothesis is in contrast with the reduction in GLP-1 levels and the normal or only slightly elevated GIP levels found in obese T2DM patients.

\section{Procedures in Bariatric/Metabolic Surgery}

A large variety of bariatric/metabolic surgical procedures which are based on restrictive or malabsorptive methods or the combination of both has been developed over time. However, in the large majority ( $>95 \%$ ) of the 468,609 bariatric/metabolic procedures performed worldwide in 2013 one of the four following interventions was used: Roux-en-Y gastric bypass (RYGB), sleeve gastrectomy, adjustable gastric banding, and biliopancreatic diversion (BPD) with or without duodenal switch [38]. All these procedures are now performed laparoscopically [39].The anatomical schemes of these four procedures are depicted in figure 2.

RYGB is a mixed technique combining both restrictive and malabsorptive mechanisms. The procedure consists of creating a small gastric pouch (15-20 ml) and bypassing the gastric remnant, duodenum, and proximal small bowel. The gastric pouch is anastomosed to the jejunum through a narrow Roux-en-Y gastrojejunal anastomosis. In order to restore the continuity of the bowel, an entero-entero anastomosis between the excluded biliopancreatic 


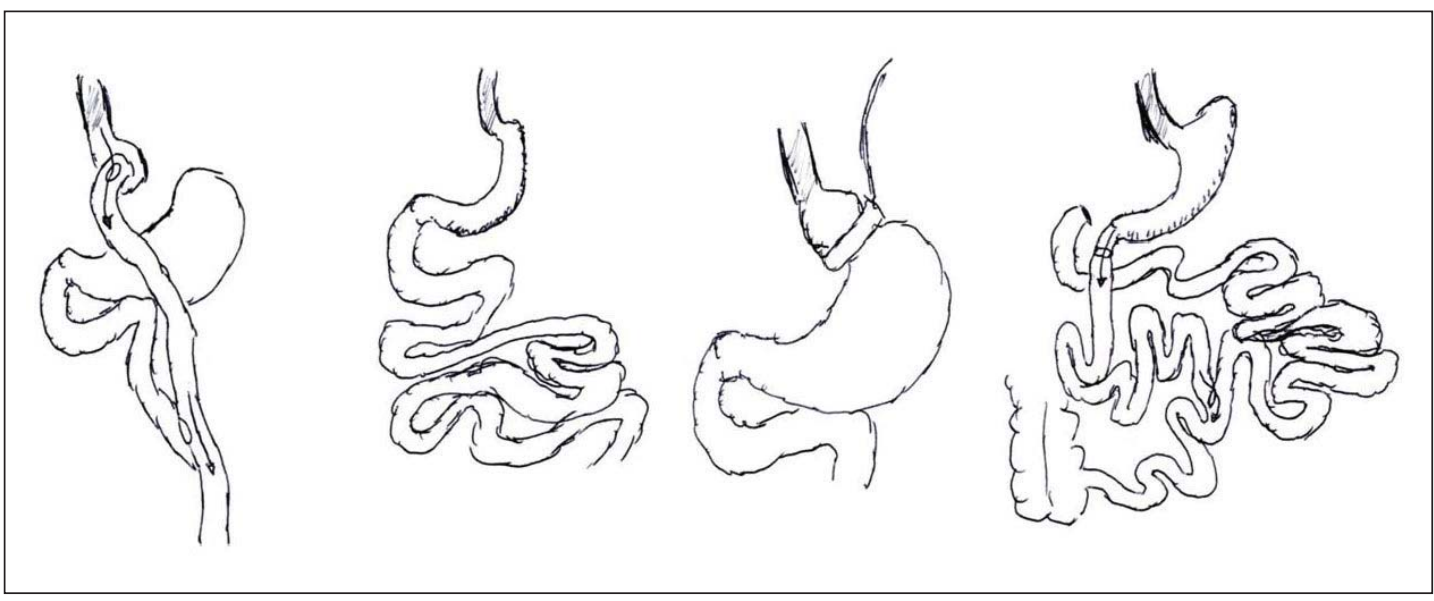

Fig. 2. Characteristics of the more diffused bariatric/metabolic surgical procedures. From left to right: RYGB, sleeve gastrectomy, adjustable gastric banding, BPD with duodenal switch.

limb and the alimentary limb is performed usually $75-100 \mathrm{~cm}$ distal from the gastrojejunostomy [40, 41].

Sleeve gastrectomy is based on the resection of the main part of fundus and corpus of the stomach, starting 2-8 $\mathrm{cm}$ proximally to the pylorus until the Angle of His. This procedure was created as the first step in a two-staged approach applied in those super-super obese patients in whom the execution of a laparoscopic BPD or RYGB was technically extremely difficult to perform in one single time. However, significant weight loss and metabolic control was observed after sleeve gastrectomy alone, and therefore the method has become very popular as an isolated procedure [39-41].

Adjustable gastric banding is a purely restrictive procedure and involves encircling the upper part of the stomach with a silicone adjustable band with an inflatable balloon at the inner surface connected with a port place subcutaneously, through which the size of the stoma can be adjusted by adding or retracting fluid [40, 41].

Finally, BPD is a primarily malabsorptive procedure consisting in a horizontal gastrectomy and an anastomosis between the remnant stomach and the distal $250 \mathrm{~cm}$ of small intestine. The bypassed duodenum, jejunum, and part of the proximal ileum carry bile and pancreatic secretions and are connected to the alimentary channel $50 \mathrm{~cm}$ proximal to the ileocecal valve. The 'common limb' is the only segment where fat and starches are absorbed [40,41].

Apart from these four major interventions, several other bariatric/metabolic procedures have been invented and proposed. Some of them disappeared because of poor results or high rates of complications and failures, and some others are still currently under scrutiny. In particular, laparoscopic greater curvature plication is a new restrictive bariatric surgery in which the greater curvature of the stomach is folded inward, creating a narrow gastric tube without involving gastric resection. Beyond significant weight loss, it seems that this technique holds metabolic effects as well $[42,43]$.

\section{How Does It Work on Type 2 Diabetes?}

As reported before, the observation that very rapid improvements in blood glucose levels and glucose homeostasis occurred within days after bariatric/metabolic surgery long before substantial weight loss, initially suggested that other mechanisms beyond weight reduction 
Fig. 3. Mechanisms involved in obesity and T2DM and the effects of metabolic surgery.

Cătoi et al.: Metabolic Mechanisms in Obesity and Type 2 Diabetes: Insights from Bariatric/Metabolic Surgery

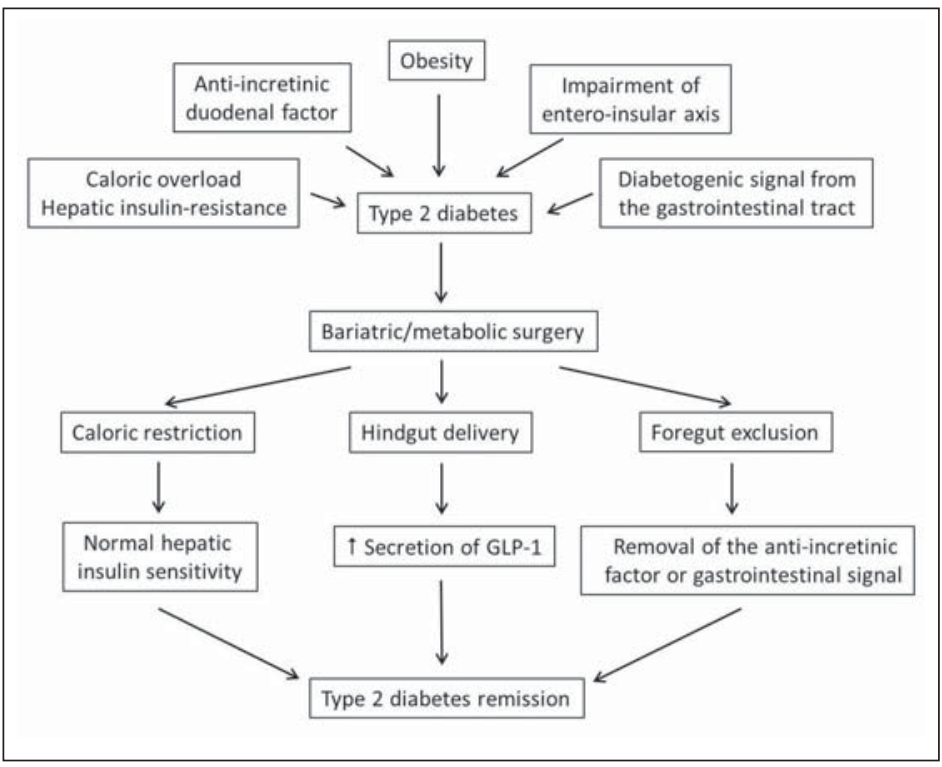

might be involved in these changes [13]. Some putative mechanisms can be related to the etiologic theories for T2DM briefly summarized in the first part of this review. In particular, attention has been focused on the role of surgical food intake restriction and on the changes in the release of gastrointestinal hormones (fig. 3).

\section{Surgical Restriction of Food Intake - Return to Normal Hepatic Insulin Sensitivity}

The importance of restriction of food intake in T2DM reversal after surgery was explained by Taylor et al. $[15,16]$ on the basis of the first steps of the disease development as suggested in their etiologic theory: the 'twin cycle hypothesis'. Indeed, the restriction of caloric intake immediately after bariatric surgery is sudden and profound. In case of rapid food shortage, the human body is forced to use other internal sources of energy: the triglyceride stores. In conditions of hypocaloric intake, ectopic fat from the liver and other depots, including pancreatic fat, are mobilized and utilized first $[13,15]$. It has been demonstrated that fasting plasma glucose improves in $81 \%$ of patients simply because of a decrease in liver fat content and normalization of hepatic insulin sensitivity, without any change in insulin resistance at the level of skeletal muscle [15]. Therefore, it could be expected that remission of T2DM after surgery might be related to removal of liver and pancreatic fat as a response to severe caloric restriction. Taylor et al. $[15,16]$ suggested therefore that acute negative calorie balance is all that is needed to reverse T2DM after bariatric/metabolic surgery.

The importance of energy restriction for the rapid improvement of glucose homeostasis in the first days after bariatric surgery is supported by the results of several studies examining the metabolic effects of nonsurgical caloric restriction. Isbell et al. [44] compared the metabolic changes occurring in the first week after RYGB with those observed in a group of matched obese patients following the same postoperative liquid diet, but not having had RYGB. With only minimal and similar levels of weight loss, a $25 \%$ improvement in insulin sensitivity was observed both in the RYGB group and in the calorie-matched nonsurgical subjects [44]. Edholm et al. [45] demonstrated a 12\% reduction of liver volume in 15 obese female candidates to laparoscopic RYGB treated preoperatively with a 4-week low-calorie diet. A mean $14.7 \%$ reduction of liver volume and a $47 \%$ reduction of liver fat content were also observed in 18 patients after a 6-week very low-energy diet [46]. Finally, Colles et al. [47] 
Cătoi et al.: Metabolic Mechanisms in Obesity and Type 2 Diabetes: Insights from Bariatric/Metabolic Surgery

found a $28.7 \%$ reduction in the liver volume after 12 weeks using the same type of diet, and they reported that $80 \%$ of the liver volume reduction occurred in the initial 2 weeks. In summary, these experimental evidences suggest that rapid improvements of glucose homeostasis and insulin resistance similar to those observed after bariatric/metabolic surgery might be obtained also in a nonsurgical setting by exposing obese patients to a similar degree of severe calorie restriction, and that these improvements are associated to significant reductions in liver volume and fat content. Additionally, Lim et al. [48] analyzed 11 people with obesity and T2DM before and after a $600 \mathrm{kcal} /$ day diet by measuring basal hepatic glucose output, hepatic and peripheral insulin sensitivity, beta cell function, and pancreas and liver triacylglycerol content. After 1 week of restricted energy intake, fasting plasma glucose normalized, insulin suppression of hepatic glucose output improved, and hepatic and pancreatic triacylglycerol content was reduced. During the same period, the first-phase insulin response increased and approached control values [48].

Therefore, normalization of both beta cell function and hepatic insulin sensitivity in T2DM can be achieved by dietary energy restriction alone, and this was associated, according to the 'twin cycle hypothesis', with decreased pancreatic and liver triacylglycerol stores [15, 16]. No other metabolic mechanisms beyond food restriction seem therefore necessary to explain the rapid postoperative metabolic improvements.

Reduction of Hyperinsulinemia - a Primary Metabolic Change That Occurs in Response to Gastric Bypass Surgery

According to the etiologic hypothesis for T2DM proposed by Pories et al. [17] that fasting hyperinsulinemia triggered by the secretion, in response to overfeeding, of a gastrointestinal diabetogenic signal is the first lesion in T2DM, the immediate normalization of fasting insulin occurring after gastric bypass could be considered the cornerstone for the rapid amelioration of T2DM. More importantly, these improvements are independent of changes in weight loss, insulin sensitivity, glucose, or free fatty acids. Indeed, the authors speculated that the exclusion of foregut leads to the removal of the hyperinsulinemic signal and consequently to less gluconeogenic substrate production and resolution of hyperglycemia [17]. This concept was supported by Reed et al. [49]. These authors studied obese non-diabetic and obese diabetic patients receiving gastric bypass and demonstrated that 1 week after surgery fasting insulin decreased to levels similar to lean controls and that T2DM remitted despite continued insulin resistance. The authors concluded that correction of hyperinsulinemia occurs mainly as the consequence of the exclusion of food from the foregut [49].

\section{The Upper Intestinal Hypothesis}

The upper intestinal hypothesis, which is known also as 'the foregut hypothesis', suggests that excluding the duodenum and proximal jejunum from contact with nutrients may avoid the over-secretion of a signal that damages glucose homeostasis, promoting decreased insulin action, impaired insulin secretion, and finally T2DM onset [50, 51]. According to this theory, an 'anti-incretinic' factor should be overproduced in the duodenum of patients with obesity and T2DM, counterbalancing the gut incretins production, which, on the other hand, is impaired in obese patients with T2DM [52]. The theory has been proposed by Rubino and Gagner [51] who consequently developed the duodenal-jejunal bypass (DJB), a metabolic surgical procedure in which the stomach was left intact, but the same amount of proximal intestine as in the classical RYGB was bypassed with a Roux-en-Y gastrojejunostomy. This procedure was applied in non-obese and obese diabetic rats, demonstrating a significant improvement in blood glucose levels, in theory without any forced food restriction or weight loss [53-55]. In a study on humans, Cohen et al. [56] reported on two patients with T2DM who underwent a DJB and experienced normoglycemia without any weight loss in either 
Cătoi et al.: Metabolic Mechanisms in Obesity and Type 2 Diabetes: Insights from Bariatric/Metabolic Surgery

patient. However, the putative diabetogenic or anti-incretin gastrointestinal hormones being the cornerstone of the Pories et al. [17] and the foregut hypothesis have not been identified so far.

\section{The Lower Intestinal Hypothesis}

The lower intestinal hypothesis, termed also as 'hindgut hypothesis', states that the rapid delivery of nutrients into the distal bowel, as a consequence of intestinal bypass (duodenum and jejunum), brings about an increased secretion of GLP-1 and other incretins, thereby leading to an improvement of glucose homeostasis $[13,57,58]$. These results are believed not to be a consequence of weight loss [24]. RYGBP and BPD are the two bariatric/metabolic procedures that more frequently lead to normal concentrations of plasma glucose, insulin and glycosylated hemoglobin in morbidly obese diabetic patients [1]. After BPD, food is delivered directly from the stomach to the ileum, and the procedure is followed by very high rates of postprandial GLP-1 secretion in plasma [58]. In RYGP, the intestinal bypass is less long, but although only a short segment of the upper intestine is excluded, there is a significant increase in the meal-stimulated GLP-1 secretion after RYGB, implying that patients who undergo this procedure show an increased incretin effect [58, 59]. An increase GLP-1 secretion after meals has never been demonstrated after adjustable gastric banding, a procedure that does not involve any modification of the gastrointestinal tract and that has a less intense effect on T2DM [60,61]. The hindgut hypothesis is further supported by the results observed after 'ileal interposition', an experimental metabolic procedure in which a segment of ileum rich in L cells producing GLP-1 is surgically interposed into the proximal intestine, near the duodenum-jejunum boundary. The exposure of the interposed ileum to ingested nutrients leads to increased GLP-1 release after meal and improves metabolic outcomes [58]. On the other hand, experimental RYGB produces the same amount of body weight and fat mass loss in GLP-1 receptor-deficient mouse, in which the endogenous GLP-1 cannot exert any effect, as in wild-type animals [62]. This new observation poses some doubts on an important individual role of GLP-1 or any other single gastrointestinal hormone in the mechanisms by which RYGB exerts its beneficial effects and suggest that more complex and integrated mechanisms are involved.

As stated before, pure restrictive bariatric procedures, such as the adjustable gastric banding, do not alter postprandial incretin levels [60,61]. Sleeve gastrectomy has been for a long-time considered a pure restrictive procedure and does not involve any change in the continuity of the gastrointestinal tract. However, sleeve gastrectomy has been shown to produce rapid improvements in glucose homeostasis and is followed by significant changes in gastrointestinal hormones similar to those observed after in RYGB: reduced ghrelin secretion and increased GLP-1 synthesis [63]. Reduced ghrelin secretion can be obviously explained by the gastric fundus resection, but the reason for increased GLP-1 production remains more elusive. A possible explanation is that GLP-1 release might be triggered not only by the direct contact of distal L cells with indigested food but also by proximal nutrient signals, e.g., increased cholecystokinin (CCK) secretion. The authors concluded that neither the upper nor the lower intestinal hypothesis can fully account for improvement in glucose homeostasis, suggesting that rather the balance between foregut (ghrelin, CCK) and hindgut hormones (GLP-1, PYY) could be the key for a better understanding of the improved glucose homeostasis [63]. In this respect, increased attention has been reserved to a possible role of bile acids (BAs) and gut microbiota.

\section{Bile Acid Changes after Bariatric Surgery}

Emerging data demonstrate that beyond their role in absorption of fats, fat-soluble vitamins and cholesterol, BAs are important key regulators of glucose and lipid metabolism 
Cătoi et al.: Metabolic Mechanisms in Obesity and Type 2 Diabetes: Insights from Bariatric/Metabolic Surgery

and energy expenditure, being involved in prevention of obesity, insulin resistance, T2DM, and the metabolic syndrome $[64,65]$. Primary BAs are produced form cholesterol in the liver. Most of them are conjugated with taurine and glycine, stored in the gallbladder, and finally secreted upon meal stimulation into the duodenum, where they mix with fatty acids and contribute to the digestion of lipids and lipid-soluble vitamins. Some primary BAs are transformed into secondary BAs by gut microbiome. In the terminal ileum BAs are absorbed, flow back to the liver and are secreted again into the bile in the enterohepatic cycle [64-66]. Known as signaling molecules, BAs mediate most of their beneficial effects on metabolism by interaction with the nuclear hormone receptor farnesoid X receptor (FXR) and the membrane receptor G-protein-coupled BA receptor 5 (TGR5) [67]. Briefly, BAs inhibit gluconeogenesis [68] and promote insulin signaling and glycogen synthase activation, thus favoring insulindependent control of glucose metabolism in the liver $[69,70]$. Through intestinal FXR, BAs stimulate postprandial fibroblast grow factor 19 (FGF19) production in the ileum, which acts on hepatocytes through specific receptors to inhibit the expression of cholesterol 7 alphahydroxylase 1 (CYP7A1), thus regulating the conversion of cholesterol into bile acids [71, 72]. BAs act also throughTGR5 and stimulate the release of GLP-1 [65, 67, 73]. This type of action of BAs fits somehow into the 'hindgut hypothesis', as it has previously been demonstrated in a canine model that both food and BAs, on their own, stimulated GLP-1 and peptide YY (PYY) responses [70].

In healthy insulin-sensitive individuals, BA levels vary as a consequence of fasting and refeeding, with an increase in response to oral glucose load [74]. However, abnormalities in BA levels have been shown in individuals with prediabetes, diabetes, and insulin resistance [75]. Ahmad et al. [76] demonstrated that, although the fasting circulating BA concentrations were not significantly different between lean and obese subjects, patients with obesity had a blunted conjugated BA excursion after a meal. In a group of morbidly obese patients with diabetes, Gerhard et al. [77] showed lower FGF19 levels and higher BA levels as compared to non-diabetic patients, and this might be most probably due to a lack of response to BAs in the liver as well as to the presence of a form of impairment in hepatic response to FGF19. Moreover, the authors underlined that the lower levels of FGF19 and the high values of BAs were present in patients with T2DM irrespective of BMI [77].

Several studies have demonstrated that BA levels increased after bariatric surgery, mainly after RYGB and sleeve gastrectomy, suggesting that elevated serum BA levels could contribute to improvements in insulin sensitivity, incretin secretion, and postprandial glycemia [70, 77-80]. However, by contrast, circulating BAs do not significantly change after gastric banding, and this may be due to the fact that in this procedure there is no rearrangement of the gastrointestinal tract [81]. The raise in BAs observed after RYGB is believed to be the result of the reduced time for the excreted BAs to mix with food, thereby leaving more free BAs for ileal re-uptake [66]. As 'digestate-free' BAs reach rapidly the distal jejunum or proximal ileum, which is where FGF19- and GLP1-producing cells are found, they may stimulate production of BAs in the liver or insulin in the pancreas [67]. After sleeve gastrectomy, there is a rapid gastric emptying that might generate an uneven distribution of gastric contents and bile secretion within the gastrointestinal tract [64]. The observation that circulating BAs increase after RYGB has brought about the assumption that they may contribute to weightindependent improvements in glucose homeostasis [82]. However, the data is conflicting. While Pournaras et al. [70] revealed that there is a significant rise in BAs and FGF19 within days after RYGB, Steinert et al. [79] showed that, while the marked increase in GLP-1 and peptide YY (PYY) secretion and the improvement in glycemic control were seen already 1 week and 3 months after surgery, basal and postprandial plasma BA concentrations increased much slower, with a significant increase only 1 year post surgery. In conclusion, some data suggest that BAs may partly explain the complex metabolic effects seen after bariatric surgery. 
Cătoi et al.: Metabolic Mechanisms in Obesity and Type 2 Diabetes: Insights from Bariatric/Metabolic Surgery

\section{Bariatric Surgery and Gut Microbiome}

Recent discoveries have revealed that the gut microbiome is involved in health and disease [83]. Human gut microbiome is very complex and seems to hold a number of cells approximately tenfold greater than the rest of the human body combined, with the greatest concentration in the ileum and colon [64, 84, 85]. Studies have shown that the human gut microbiome is composed primarily of the phyla Bacteroidetes and Firmicutes. Gut microbiota interacts with the host in several ways including i) modulation of the inflammatory host response; ii) synthesis of small molecules and proteins that are taken up by the host; and iii) changes in the amount of available energy in the diet [85]. The gut microbiome has emerged as an important contributor to the obesity and T2DM epidemic and is proposed to act by increasing energy harvest from the diet, by altering metabolic and appetite signaling pathways and by inducing chronic inflammation [86-88]. However, the precise pathophysiology of gut microbiome in obesity remains only partly understood [86]. Some findings have shown that in the gut of obese humans and mice, the ratio of Firmicutes to Bacteroidetes is elevated and falls with weight loss [89].

Bariatric surgery not only improves hormonal and inflammatory status but also induces numerous changes in the digestive tract that might account for some modifications of microbiome ecology [90]. Thereby, several studies have examined the role of the distal gut microbiome in obesity and the changes that occur after RYGB in humans and rodents [85]. One study showed that the transfer of the microbiota from RYGB-treated mice to germ-free mice resulted in weight loss, indicating that changes in the gastrointestinal microbiome following surgery may contribute to weight loss [91]. On the other hand, changes in the composition of gut microbiome with important effects on the metabolism have been reported after RYGB. More precisely, a decrease in the Firmicutes:Bacteroides ratio $[92,93]$ and an increase in the bile-tolerant Proteobacteria [93-95] have been described. These changes might be partly explained by the changes in the diet [96]. Moreover, there seems to be a complex relationship between gut microbiome and BAs. The presence of a higher amount of free BAs in the lower intestine creates an environment encouraging the growth of the Proteobacter phylum [97]. In turn, Proteobacter causes a decrease in secondary BAs, leading to increased serum primary BA levels [97]. Taken together, these data suggests that the rerouting of the intestinal anatomy, and the changes of gut microbiome and BA levels that are brought about, may improve systemic metabolism through complex mechanisms.

\section{Conclusions}

A large and exhaustive meta-analysis recently confirmed that bariatric/metabolic surgery has a greater weight loss effect and a higher rate of T2DM remission than conventional therapy $[12,98]$. Although metabolic surgery involves an initial financial investment, the use of this type of interventions in T2DM obese patients, who otherwise imply important health costs, may be associated with important savings in a relatively short period of time [99], and the costs of antidiabetic drugs 2 years after surgery is estimated to be $79 \%$ lower than before the operation [100].

Most of the benefits of bariatric/surgery can be attributed to weight loss, especially in the long term. However, some important clinical improvements, in particular in respect to glucose homeostasis and T2DM occur independently from weight loss levels, suggesting that alternative mechanisms may play a role. The uncertainty that we still have about what these mechanisms are and to which extent they contribute to the effects detected is reflected in our incomplete understanding about the etiologic mechanisms of T2DM in obesity. Surgical food 
restriction is certainly important, but, depending on the type of rearrangements of the gastrointestinal tract, other weight-independent antidiabetic mechanisms can be involved, such as the incretinic/anti-incretinic system and possibly even more other pathways. At the end, bariatric/metabolic surgery remains a complex subject of research, and the elucidation of its mechanisms of action can help to clarify the still elusive pathophysiology of T2DM in obesity.

\section{Acknowledgements}

This paper was published under the frame of European Social Fund, Human Resources Development Operational Programme 2007-2013, project no. POSDRU/159/1.5/S/138776.

\section{Disclosure Statement}

The authors have no conflict of interest to report.

\section{References}

1 Buchwald H, Avidor Y, Braunwald E, Jensen MD, Pories W, Fahrbach K, Schoelles K: Bariatric surgery: a systematic review and meta-analysis. JAMA 2004;292:1724-1737.

2 Farag YMK, Gaballa MR: Diabesity: an overview of a rising epidemic. Nephrol Dial Transplant 2011;26:28-35.

3 Copăescu C: Metabolic surgery. Acta Endocrinol (Buc), 2013;IX:273-278.

4 Rubino F: From bariatric to metabolic surgery: definition of a new discipline and implications for clinical practice. Curr Atheroscler Rep 2013;15:369.

5 Buchwald H, Varco RL: Metabolic Surgery. New York, Grune and Stratton, 1978.

6 Rubino F, Schauer PR, Kaplan LM, Cummings DE: Metabolic surgery to treat type 2 diabetes: clinical outcomes and mechanisms of action. Annu Rev Med 2010;61:393-411.

7 Buchwald H, Estok R, Fahrbach K, et al: Weight and type 2 diabetes after bariatric surgery: systematic review and meta-analysis. Am J Med 2009;122:248-256.

8 Dixon JB, O'Brien PE, Playfair J, et al: Adjustable gastric banding and conventional therapy for type 2 diabetes: a randomized controlled trial. JAMA 2008;299:316-323.

9 Schauer PR, Kashyap SR, Wolski K, et al: Bariatric surgery versus intensive medical therapy in obese patients with diabetes. N Engl J Med 2012;26:366:1567-1576.

10 Mingrone G, Panunzi S, De Gaetano A, et al: Bariatric surgery versus conventional medical therapy for type 2 diabetes. N Engl J Med 2012;26:366:1577-1585.

11 Ikramuddin S, Korner J, Lee WJ, et al: Roux-en-Y gastric bypass vs intensive medical management for the control of type 2 diabetes, hypertension, and hyperlipidemia. The Diabetes Surgery Study randomized clinical trial. JAMA 2013;309:2240-2249.

12 Müller-Stich BP, Senft JD, Warshkow R, et al: Surgical versus medical treatment of type 2 diabetes mellitus in nonseverely obese patients: a systematic review and meta-analysis. Ann Surg 2015;261:421-429.

13 Knop F, Taylor R: Mechanism of metabolic advantages after bariatric surgery. Diabetes Care 2013;36(suppl 2):S287-291.

14 Lazar MA: How obesity causes diabetes: not a tall tale. Science 2005;307:373-375.

15 Taylor R: Type 2 diabetes: etiology and reversibility. Diabetes Care 2013;36:1047-1055.

16 Taylor R: Pathogenesis of type 2 diabetes: tracing the reverse route from cure to cause. Diabetologia 2008; 51 : 1781-1789.

17 Pories WJ, Dohm GL: Diabetes: have we got it all wrong? Hyperinsulinism as the culprit: surgery provides the evidence. Diabetes Care 2012;35:2438-2442.

18 Turner RC, Cull CA, Frighi V, Holman RR; UK Prospective Diabetes Study (UKPDS) Group: Glycemic control with diet, sulfonylurea, metformin, or insulin in patients with type 2 diabetes mellitus: progressive requirement for multiple therapies (UKPDS 49). JAMA 1999;281:2005-2012.

19 Petersen KF, Dufour S, Savage DB, et al: The role of skeletal muscle insulin resistance in the pathogenesis of the metabolic syndrome. Proc Natl Acad Sci U S A 2007;104:12587-12594.

20 Taylor R: Insulin resistance and type 2 diabetes. Diabetes 2012;61:778-779.

21 Unger RH, Eisentraut AM: Entero-insular axis. Arch Intern Med 1969;123:261-266.

22 Creutzfeldt W: The incretin concept today. Diabetologia 1979;16:75-85.

23 Patriti A, Facchiano E, Sanna A, Gullà N, Donini A: The enteroinsular axis and the recovery from type 2 diabetes after bariatric surgery. Obes Surg 2004;14:840-848. 


\begin{tabular}{l|l}
\hline Obes Facts 2015;8:350-363 \\
\hline DOI: 10.1159/000441259 & $\begin{array}{l}\text { @ 2015 S. Karger GmbH, Freiburg } \\
\text { www.karger.com/ofa }\end{array}$ \\
\hline
\end{tabular}

Cătoi et al.: Metabolic Mechanisms in Obesity and Type 2 Diabetes: Insights from Bariatric/Metabolic Surgery

24 Frezza E: Are we closer to finding the treatment for type 2 diabetes mellitus in morbid obesity? Are the incretins the key to success? Obes Surg 2004;14:999-1005.

25 Gumbs AA, Modlin IM, Ballantyne GH: Changes in insulin resistance following bariatric surgery: role of caloric restriction and weight loss. Obes Surg 2005; 15:462-473.

26 Ashrafian H, le Roux CW: Metabolic surgery and gut hormones - a review of bariatric entero-humoral modulation. Physiol Behav 2009;97:620-631.

27 Perfetti R, Zhou J, Doyle ME, Egan JM: Glucagon-like peptide-I induces cell proliferation and pancreaticduodenum homeobox-1 expression and increases endocrine cell mass in the pancreas of old, glucose-intolerant rats. Endocrinology 2000;141:4600-4605.

28 Drucker DJ: Glucagon-like peptides: regulators of cell proliferation, differentiation, and apoptosis. Mol Endocrinol 2003;17:161-171.

29 Mingrone G: Role of the incretin system in the remission of type 2 diabetes following bariatric surgery. Nutr Metab Cardiovasc Dis 2008;18:574-579.

30 Baggio LL, Drucker DJ: Biology of incretins: GLP-1 and GIP. Gastroenterology 2007;132:2131-2157.

31 Roberge JN, Gronau KA, Brubaker PL: Gastrin-releasing peptide is a novel mediator of proximal nutrientinduced proglucagon-derived peptide secretion from the distal gut. Endocrinology 1996;137:2383-2388.

32 Lugari R, Dei Cas A, Ugolotti D, et al: Evidence for early impaired of glucagon-like peptide 1-induced insulin secretin in human type 2 (non insulin-dependent) diabetes. Horm Metab Res 2002;34:150-154.

33 Mannucci E, Ognibene A, Cremasco F, et al: Glucagon-like peptide (GLP)-1 and leptin concentrations in obese patients with type 2 diabetes mellitus. Diab Med 2000;17:713-719.

34 Vilsbøll T, Krarup T, Deacon CF, Madsbad S, Holst JJ: Reduced postprandial concentrations of intact biologically active glucagon-like peptide 1 in type 2 diabetic patients. Diabetes 2001;50:609-613.

35 Fetner R, McGinty J, Russell C, Pi-Sunyer FX, Laferrère B: Incretins, diabetes, and bariatric surgery: a review. Surg Obes Relat Dis 2005;16:589-597.

36 Preitner F, Ibberson M, Franklin I, et al: Glucoincretins control insulin secretion at multiple levels as revealed in mice lacking GLP-1 and GIP receptors. J Clin Invest 2004;113:635-645.

37 Pories WJ: Why does the gastric bypass control type 2 diabetes mellitus? Obes Surg 2002;2:303-313.

38 Pories WJ, Swanson MS, MacDonald KG, et al: Who would have thought it? An operation proves to be the most effective therapy for adult-onset diabetes mellitus. Ann Surg 1995;222:339-350.

39 Angrisani L, Santonicola A, Iovino P, et al: Bariatric surgery worldwide 2013. Obes Surg 2015;25:1822-1832.

40 Rubino F, Schauer PR, Kaplan LM, Cummings DE: Metabolic surgery to treat type 2 diabetes: clinical outcomes and mechanisms of action. Annu Rev Med 2010;61:393-411.

41 Gass M, Beglinger C, Peterli R. Metabolic surgery - principles and current concepts. Langenbecks Arch Surg 2011;396:949-972.

42 Copăescu C: Laparoscopic gastric plication at the greater curvature (for treatment of morbid obesity) Chirurgia (Bucur) 2011;106:91-97.

43 Bradnova O, Kyrou I, Hainer V, et al: Laparoscopic greater curvature plication in morbidly obese women with type 2 diabetes: effects on glucose homeostasis, postprandial triglyceridemia and selected gut hormones. Obes Surg 2014;24:718-726.

44 Isbell JM, Tamboli RA, Hansen EN, et al: The importance of caloric restriction in the early improvements in insulin sensitivity after Roux-en-Y gastric bypass surgery. Diabetes Care 2010;33:1438-1442.

45 Edholm D, Kullberg J, Haenni A, et al: Preoperative 4-week low-calorie diet reduces liver volume and intrahepatic fat, and facilitates laparoscopic gastric bypass in morbidly obese. Obes Surg 2011;21:345-350.

46 Lewis MC, Phillips ML, Slavotinek JP, et al: Change in liver size and fat content after treatment with Optifast ${ }^{\circledR}$ very low calorie diet. Obes Surg 2006;16:697-701.

47 Colles SL, Dixon JB, Marks P, Strauss BJ, O'Brien PE: Preoperative weight loss with a very-low-energy diet: quantitation of changes in liver and abdominal fat by serial imaging. Am J Clin Nutr 2006;84:304-311.

48 Lim EL, Hollingsworth KG, Aribisala BS, Chen MJ, Mathers JC, Taylor R: Reversal of type 2 diabetes: normalisation of beta cell function in association with decreased pancreas and liver triacylglycerol. Diabetologia 2011; 54:2506-2514.

49 Reed MA, Pories WJ, Chapman W, et al: Roux-en-Y gastric bypass corrects hyperinsulinemia implications for the remission of type 2 diabetes. J Clin Endocrinol Metab 2011;96:2525-2531.

50 Pories WJ, Albrecht RJ: Etiology of type II diabetes mellitus: role of the foregut. World J Surg 2001;25:527-531.

51 Rubino F, Gagner M: Potential of surgery for curing type 2 diabetes mellitus. Ann Surg 2002;236:554-559.

52 Rubino F, Forgione A, Cummings DE, et al: The mechanism of diabetes control after gastrointestinal bypass surgery reveals a role of the proximal small intestine in the pathophysiology of type 2 diabetes. Ann Surg 2006; 244:741-749.

53 Rubino F, Marescaux J: Effect of duodenal-jejunal exclusion in a nonobese animal model of type 2 diabetes: a new perspective for an old disease. Ann Surg 2004;239:1-11.

54 Rubino F, Zizzari P, Tomasetto C, et al: The role of the small bowel in the regulation of circulating ghrelin levels and food intake in the obese Zucker rat. Endocrinology 2005;146:1745-1751.

55 Pacheco D, de Luis DA, Romero A, et al: The effects of duodenal-jejunal exclusion on hormonal regulation of glucose metabolism in Goto-Kakizaki rats. Am J Surg 2007;194:221-224.

56 Cohen RV, Schiavon CA, Pinheiro JS, Correa J Luiz, Rubino F: Duodenal-jejunal bypass for the treatment of type 2 diabetes in patients with BMI 22-34: a report of two cases. Surg Obes Relat Dis 2007;3:195-197. 
57 Dixon JB: Obesity and diabetes: the impact of bariatric surgery on type-2 diabetes. World J Surg 2009;33: 2014-2021.

58 Thaler JP, Cummings DE: Minireview: hormonal and metabolic mechanisms of diabetes remission after gastrointestinal surgery. Endocrinology 2009;150:2518-2525.

59 Laferrère B, Heshka S, Wang K, et al: Incretin levels and effect are markedly enhanced 1 month after Roux-enY gastric bypass surgery in obese patients with type 2 diabetes. Diabetes Care 2007;30:1709-1716.

60 Usinger L, Hansen KB, Kristiansen VB, Larsen S, Holst JJ, Knop FK: Gastric emptying of orally administered glucose solutions and incretin hormone responses are unaffected by laparoscopic adjustable gastric banding. Obes Surg 2011;21:625-632.

61 Korner J, Bessler M, Inabnet W, Taveras C, Holst JJ: Exaggerated glucagon-like peptide-1 and blunted glucosedependent insulinotropic peptide secretion are associated with Roux-en-Y gastric bypass but not adjustable gastric banding. Surg Obes Relat Dis 2007;3:597-601.

62 Ye J, Hao Z, Mumphrey MB, et al: GLP-1 receptor signaling is not required for reduced body weight after RYGB in rodents. Am J Physiol Regul Integr Comp Physiol 2014;306:R352-362.

63 Peterli R, Steinert RE, Woelnerhanssen B, et al: Metabolic and hormonal changes after laparoscopic Roux-enY gastric bypass and sleeve gastrectomy: a randomized, prospective trial. Obes Surg 2012;22:740-748.

64 Dixon JB, Lambert EA, Lambert GW: Neuroendocrine adaptations to bariatric surgery. Mol Cell Endocrinol 2015; doi: 10.1016/j.mce.2015.05.033.

65 Ma H, Patti ME: Bile acids, obesity, and the metabolic syndrome. Best Pract Res Clin Gastroenterol 2014;28: 573-583.

66 Sweeney TE, Morton JM: Metabolic surgery: action via hormonal milieu changes, changes in bile acids or gut microbiota? A summary of the literature. Best Pract Res Clin Gastroenterol 2014;28:727-740.

67 Argyropoulos G: Bariatric surgery: prevalence, predictors, and mechanisms of diabetes remission. Curr Diab Rep 2015;15:15.

68 Thomas C, Pellicciari R, Pruzanski M, Auwerx J, Schoonjans K: Targeting bile-acid signalling for metabolic diseases. Nat Rev Drug Discov 2008;7:678-693.

69 Han SI, Studer E, Gupta S, et al: Bile acids enhance the activity of the insulin receptor and glycogen synthase in primary rodent hepatocytes. Hepatology 2004;39:456-463.

70 Pournaras DJ, Glicksman C, Vincent RP, et al: The role of bile after Roux-en-Y gastric bypass in promoting weight loss and improving glycaemic control. Endocrinology 2012;153:3613-3619.

71 Chiang JY: Bile acids: regulation of synthesis. J Lipid Res 2009;50:1955-1966.

72 Lee FY, Lee H, Hubbert ML, Edwards PA, Zhang Y: FXR, a multipurpose nuclear receptor. Trends Biochem Sci 2006;31:572-580.

73 Pols TW, Noriega LG, Nomura M, Auwerx J, Schoonjans K: The bile acid membrane receptor TGR5 as an emerging target in metabolism and inflammation. J Hepatol 2011;54:1263-1272.

74 Shaham O, Wei R, Wang TJ, et al: Metabolic profiling of the human response to a glucose challenge reveals distinct axes of insulin sensitivity. Mol Syst Biol 2008;4:214.

75 Haeusler RA, Astiarraga B, Camastra S, Accili D, Ferrannini E: Human insulin resistance is associated with increased plasma levels of $12 \alpha$-hydroxylated bile acids. Diabetes 2013;62:4184-4191.

76 Ahmad NN, Pfalzer A, Kaplan LM: Roux-en-Y gastric bypass normalizes the blunted postprandial bile acid excursion associated with obesity. Int J Obes (Lond) 2013;37:1553-1559.

77 Gerhard GS, Styer AM, Wood GC, et al: A role for fibroblast growth factor 19 and bile acids in diabetes remission after Roux-en-Y gastric bypass. Diabetes Care 2013;36:1859-1864.

78 Penney NC, Kinross JM, Newton RC, Purkayastha S: The role of bile acids in reducing the metabolic complications of obesity after bariatric surgery: A systematic review. Int J Obes 2015; doi: 10.1038/ijo.2015.115.

79 Steinert RE, Peterli R, Keller S, et al: Bile acids and gut peptide secretion after bariatric surgery: a 1-year prospective randomized pilot trial. Obesity (Silver Spring) 2013;21:E660-E668.

80 Patti ME, Houten SM, Bianco AC, et al: Serum bile acids are higher in humans with prior gastric bypass: potential contribution to improved glucose and lipid metabolism. Obesity (Silver Spring) 2009;17:16711677.

81 Kohli R, Bradley D, Setchell KD, et al: Weight loss induced by Roux-en-Y gastric bypass but not laparoscopic adjustable gastric banding increases circulating bile acids. J Clin Endocrinol Metab 2013;98:E708-E712.

82 Laferrere B: Gut feelings about diabetes. Endocrinol Nutr. 2012;59:254-260.

83 Kerr CA, Grice DM, Tran CD, et al: Early life events influence whole-of-life metabolic health via gut microflora and gut permeability. Crit Rev Microbiol 2015;41:326-240.

84 Luckey TD: Introduction to the ecology of the intestinal flora. Am J Clin Nutr 1970;23:1430--1432.

85 Sweeney TE, Morton JM: The human gut microbiome: a review of the effect of obesity and surgically induced weight loss. JAMA Surg 2013;148:563-569.

86 Kootte RS, Vrieze A, Holleman F, et al: The therapeutic potential of manipulating gut microbiota in obesity and type 2 diabetes mellitus. Diabetes Obes Metab 2012;14:112-120.

87 Tremaroli V, Backhed F: Functional interactions between the gut microbiota and host metabolism. Nature 2012;489:242-249.

88 Cox, AJ, West NP, Cripps AW: Obesity, inflammation, and the gut microbiota. Lancet Diabetes Endocrinol 2015; 3:207-215. 
Cătoi et al.: Metabolic Mechanisms in Obesity and Type 2 Diabetes: Insights from Bariatric/Metabolic Surgery

89 Ley RE, Turnbaugh PJ, Klein S, Gordon JI: Microbial ecology: human gut microbes associated with obesity. Nature 2006;444:1022-1023.

90 Aron-Wisnewsky J, Doré J, Clement K: The importance of the gut microbiota after bariatric surgery. Nat Rev Gastroenterol Hepatol 2012;9:590-598.

91 Liou AP, Paziuk M, Luevano JM Jr, et al: Conserved shifts in the gut microbiota due to gastric bypass reduce host weight and adiposity. Sci Transl Med 2013;5:178ra141.

92 Furet JP, Kong LC, Tap J, et al: Differential adaptation of human gut microbiota to bariatric surgery-induced weight loss: links with metabolic and low-grade inflammation markers. Diabetes 2010;59:3049-3057.

93 Zhang H, DiBaise JK, Zuccolo A, et al: Human gut microbiota in obesity and after gastric bypass. Proc Natl Acad Sci U S A 2009;106:2365-2370.

94 Graessler J, Qin Y, Zhong H, et al: Metagenomic sequencing of the human gut microbiome before and after bariatric surgery in obese patients with type 2 diabetes: correlation with inflammatory and metabolic parameters. Pharmacogenomics J 2013;13:514-522.

95 Osto M, Abegg K, Bueter M, et al: Roux-en-Y gastric bypass surgery in rats alters gut microbiota profile along the intestine. Physiol Behav 2013;119:92-96.

96 David LA, Maurice CF, Carmody RN, et al: Diet rapidly and reproducibly alters the human gut microbiome. Nature 2014;505:559-563.

97 Vrieze A, Out C, Fuentes S, et al: Impact of oral vancomycin on gut microbiota, bile acid metabolism, and insulin sensitivity. J Hepatol 2014;60:824-831.

98 Ribaric G, Buchwald JN, McGlennon TW: Diabetes and weight in comparative studies of bariatric surgery vs conventional medical therapy: a systematic review and meta-analysis. Obes Surg 2014;24:437-455.

99 Terranova L, Busetto L, Vestri A, Zappa MA: Bariatric surgery: cost-effectiveness and budget impact. Obes Surg 2012;22:646-653.

100 Sane T, Pekkarinen T, Jaser N, Juuti A, Virtanen L, Leivonen M: The effect of gastric sleeve and bypass surgery on the use and costs of medications for type 2 diabetes. Duodecim 2013;129:1590-1597. 